OPEN ACCESS

Edited by:

Xiaoquan Rao,

Case Western Reserve University,

United States

Reviewed by:

Alok Agrawal,

East Tennessee State University,

United States

Hongkuan Fan,

Medical University of South Carolina,

United States

Daping Fan,

University of South Carolina

United States

*Correspondence:

Liwu Li

Iwli@vt.edu

Specialty section: This article was submitted to

Molecular Innate Immunity,

a section of the journal

Frontiers in Immunology

Received: 27 April 2018 Accepted: 12 September 2018

Published: 04 October 2018

Citation:

Zhang Y, Geng S, Prasad GL and Li L (2018) Suppression of Neutrophil Antimicrobial Functions by Total Particulate Matter From Cigarette Smoke. Front. Immunol. 9:2274. doi: 10.3389/fimmu.2018.02274

\section{Suppression of Neutrophil Antimicrobial Functions by Total Particulate Matter From Cigarette Smoke}

\author{
Yao Zhang ${ }^{1}$, Shuo Geng ${ }^{1}$, G. L. Prasad ${ }^{2}$ and Liwu $L^{1 *}$ \\ ${ }^{1}$ Department of Biological Sciences, Virginia Tech, Blacksburg, VA, United States, ${ }^{2}$ RAI Services Company, Winston-Salem, \\ NC, United States
}

Chronic cigarette smoking is widely known to alter immune functions and compromise host defense against microbial infection. Neutrophils play an essential role in the immune defense against microbial pathogens and also participate in the development of the inflammatory responses. However, there is limited information about the effects of cigarette smoking on neutrophil response. In this study, cultured bone marrow neutrophils were exposed to total particulate matter (TPM) from cigarette smoke. We found that TPM not only reduced LPS-induced TNF $\alpha$ production, but also suppressed neutrophil bactericidal activity. We also observed that TPM priming reduced the expression of NADPH oxidase component gp91 and iNOS, molecules important for bacterial killing. Mechanistically, we documented that TPM-primed neutrophils have reduced STAT1 activation following subsequent LPS challenge. STAT1 is a key transcription factor responsible for the expression of inflammatory genes as well as gp91 and iNOS. Collectively, reduced STAT1 activation and reduced NADPH oxidase/iNOS may potentially explain the compromised anti-microbial function of TPM-programmed neutrophils. Taken together, our findings reveal that the key innate immune neutrophil is subject to reprogramming by smoking to adopt an immune-suppressed state, potentially responsible for chronic smoking-mediated immunosuppression.

\section{Keywords: neutrophil programming, TPM, immune-modulation, NADPH oxidase, inflammation}

\section{INTRODUCTION}

Cigarette smoking has been well recognized as a major risk factor for lung diseases such as chronic obstructive pulmonary disease (COPD), pneumonia, asthma, and lung cancer $(1,2)$. The pathogenesis of smoking-associated lung diseases is highly complex and potentially involves altered innate and adaptive immune responses. Alterations in adaptive immunity and subsequent inflammatory complications may underlie chronic airway remodeling associated with smokingmediated COPD and cancer (3-5). In addition, smoking is also reported to directly affect the function of innate immune cells such as macrophages, dendritic cells, natural killer cells, and neutrophils, potentially contributing to compromised innate defense and execerbated lung infection (6-10).

Although past studies provided compelling evidence with regard to smoking-related alteration of adaptive immune cells, relatively few mechanistic studies are available in the context of altered 
innate immune cell functions related with smoking. Alveolar macrophages from cigarette smokers exhibit reduced phagocytic activity toward airway pathogens (11-13). In addition, compromised dendritic cells and natural killer cells may also collectively contribute to compromised bacterial killing function $(7,14)$. Alterations in neutrophil functions in smokers have been largely associated with increased inflammation and tissue damage $(15,16)$. Cigarette smoke-activated neutrophils from smokers congregate in lung vasculature, causing elevated release of inflammatory mediators such as metalloproteinase-9 (17) and Leukotriene B4 (LTB4) $(18,19)$. These mediators subsequently cause oxidative and proteolytic tissue damage, aggravating immune dysregulation leading to chronic lung disease. However, it is less clear whether cigarette smoking or exposure to cigarette smoke may directly impair the bacterial-killing function of neutrophils.

To address this question, we hereby examined whether neutrophil-mediated bacterial killing is impacted by total particulate matter (TPM) from cigarette smoke. We have compared the bacterial killing potential of naive and TPMchallenged primary murine neutrophils and have investigated underlying mechanisms. We report that TPM treatment significantly compromises bacterial killing activity of neutrophils. Mechanistically, NADPH oxidase as well as inducible nitric oxide synthase (iNOS) are important in mediating the bacterial-killing activity. We observed that TPM-treated neutrophils fail to induce gp90 and iNOS expression following LPS challenge. STAT1 is a critical transcription factor involved in the expression of both gp90 and iNOS $(20,21)$. We observed that TPM treatment compromises the STAT1 activation induced by LPS. Taken together, our study reveals novel mechanisms for TPM-mediated neutrophil reprogramming with reduced bacterial killing activity, in that TPM reduces STAT1 mediated expression of key antibacterial molecules such as gp90 and iNOS.

\section{MATERIALS AND METHODS}

\section{Mice}

Wild type (WT) C57BL/6 mice were purchased from the Charles River laboratory. All mice were housed under specific pathogenfree conditions and bred and maintained in the animal facility at Virginia Tech with the approved Animal Care and Use Committee protocol. Groups of 6-8 weeks old mice were used in all experiments.

\section{Reagents}

LPS (Escherichia coli 0111:B4) was obtained from Sigma Aldrich. TPM from 3R4F reference cigarettes was prepared as described previously and provided by R.J. Reynolds Tobacco Company, Winston-Salem, NC (22). FITC conjugated antimouse Ly6G antibody, PE/Cy7 conjugated anti-mouse TNFa antibody, APC/Cy7 and PE conjugated anti-mouse/human CD11b antibody, were from Biolegend (San Diego, CA).

\section{TPM Preparation}

TPM from 3R4F reference cigarettes (University of Kentucky) was prepared at Labstat International, Kitchner, Canada as described previously (22). The reference cigarettes were smoked using the standard ISO method (35-60-2, puff volume in $\mathrm{mL}$, inter puff interval in seconds and puff duration in seconds). The particular matter was collected on a Cambridge filter pad and dissolved in DMSO. The nicotine content of the TPM was quantified, and expressed in $\mu \mathrm{g} / \mathrm{mL}$. We used this measure, termed equi-nicotine units, to compare the effect of TPM and to normalize the results.

\section{Neutrophil Isolation and Culture}

Neutrophils from bone marrow were isolated from the tibias and femurs of wild type (WT) mice, purified over a Percoll gradient of 82,65 , and 55\%. Neutrophils were collected at the $65-82 \%$ interface, and cultured in completed RPMI (10\% FBS, $10 \mathrm{mM}$ HEPES, $1 \%$ penicillin/streptomycin, Lglutamine $2 \mathrm{mM}$, and $10 \mathrm{ng} / \mathrm{ml} \mathrm{G}-\mathrm{CSF}$ ) (23). The purity and viability of neutrophils post purification and before the start of the experiment were $>90 \%$ as measured by flow cytometry (Figure 1A).

\section{Flow Cytometry}

Single-cell suspensions were prepared from bone marrow (23). Prior to intracellular staining for cytokines, cells were cultured in vitro for $4 \mathrm{~h}$ in RPMI completed medium with GolgiStop ${ }^{\mathrm{TM}}$ (BD Biosciences, San Jose, CA). Then the cells were fixed after surface marker staining, permeabilized and stained with anti-TNFa, according to the manufacturer's instructions (BD Biosciences). Samples were analyzed with a FACS Canto II (BD Biosciences). FACS plots shown were analyzed with FlowJo (Ashland, OR).

\section{In vitro Bacterial Killing Assay}

Fresh overnight cultures of E.coli were resuspended in HBSS and opsonized with $20 \%$ mouse serum. Purified neutrophils were cultured and treated with LPS $(100 \mathrm{ng} / \mathrm{ml})$ or TPM $(70 \mathrm{ng} / \mathrm{ml}$ of equi-nicotine units) overnight. The primed neutrophils and opsonized bacteria were incubated together at 1:5 ratio for $1 \mathrm{~h}$. Following the incubation, neutrophils were lysed by $0.05 \%$ triton and diluted aliquots were spread on an LB plate. Bacterial colonies were counted the next day (24). Bacterial suspension without any cells were used as input control. Relative killing rates were calculated by (input CFU-leftover $\mathrm{CFU})$ /input CFU.

\section{NADPH Measurement}

Purified neutrophils were cultured and treated with LPS ( $100 \mathrm{ng} / \mathrm{ml})$ and/or TPM (70 $\mathrm{ng} / \mathrm{ml}$ equi-nicotine units) overnight, followed with or without stimulation of PMA $(20 \mathrm{ng} / \mathrm{ml})$ for $15 \mathrm{~min}$, then subjected to NADP/NADPH-Glo ${ }^{\mathrm{TM}}$ assay, according to the manufacturer's instructions (Promega, Madison, WI).

\section{Protein Extraction and Western Blot Analyses}

Cells were washed with PBS and harvested in $1 \times$ SDS lysis buffer containing protease inhibitor cocktail and subjected to SDS-PAGE (25). Seperated proteins were transferred to an 

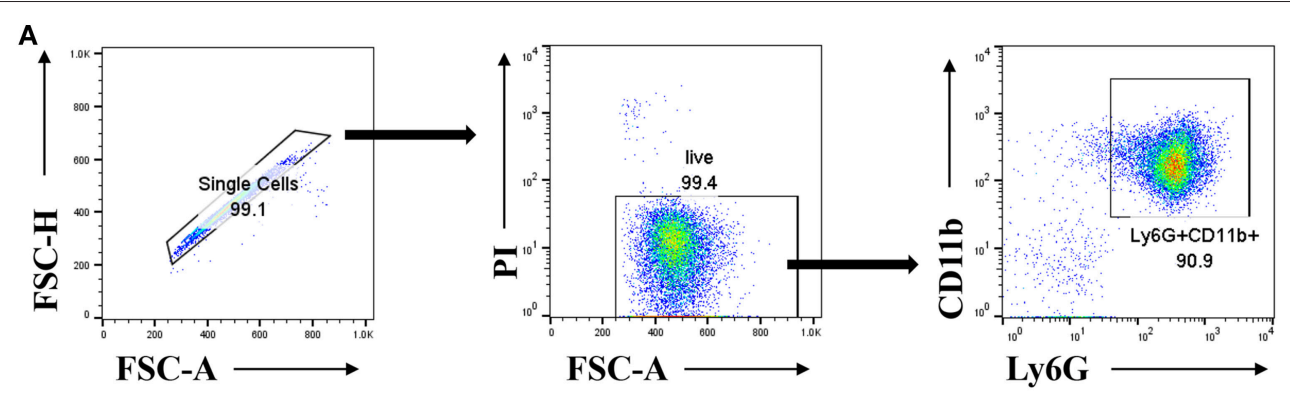

TPM

B (equi-nicotine units, $\mathrm{ng} / \mathrm{mL}$ )
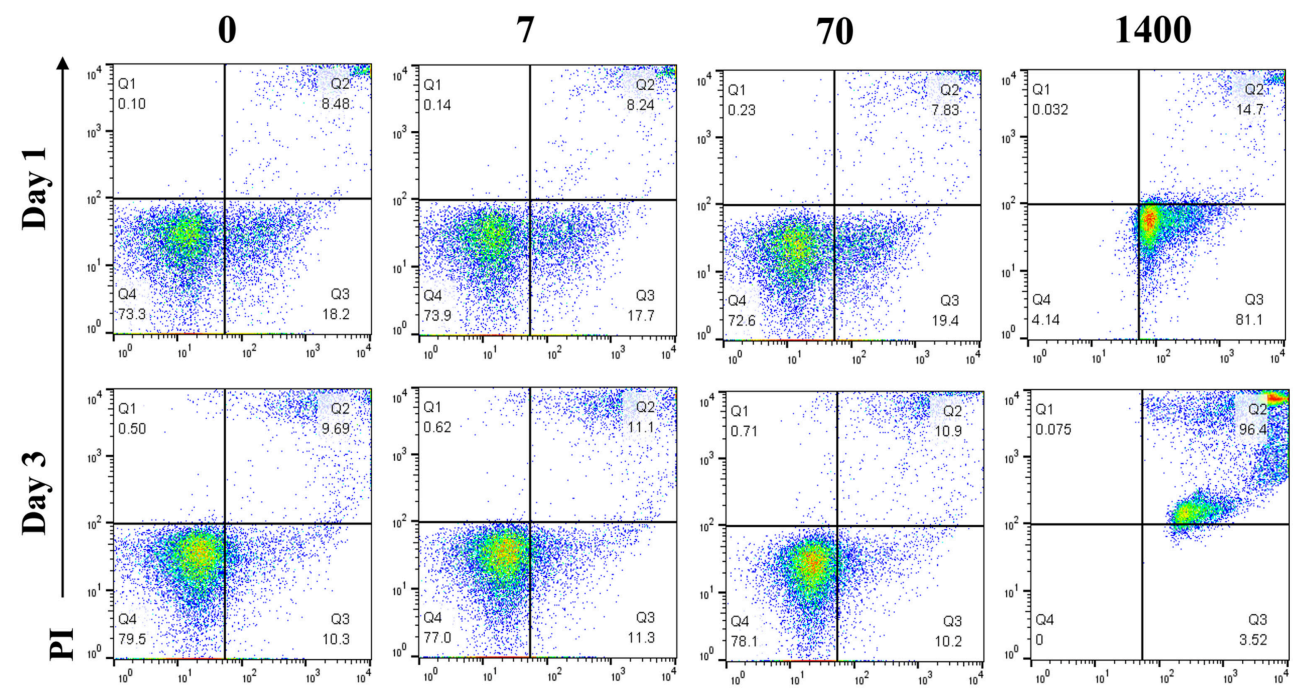

Annexin V

FIGURE 1 | Lower dosages of TPM do not increase neutrophil cell death. (A) Reprensentive flow cytometry analysis of viability and purity of isolated neutrophils from mouse bone marrow through percoll gradient as described in the Materials and Methods section. (B) Purified neutrophils were cultured in completed RPMI medium. Neutrophils were treated with different concentrations ( $\mathrm{ng} / \mathrm{ml}$ equi-nicotine units) of TPM for 3 days. Flow cytometry analyses were performed to measure cell death as described in the Materials and Methods section. Representative results from three experiments are shown. Numbers on dot plots represent the percentage of apoptotic neutrophils cells stained by $\mathrm{Pl}$ and annexin V.

immunoblot PVDF membrane (BioRad). Western blot analyses were performed with specified antibodies as shown in reagents above.

\section{ELISA}

Purified neutrophils were pre-treated without or with autophagy inhibitor Spautin1 $30 \mathrm{~min}$ before stimulation with LPS ( $100 \mathrm{ng} / \mathrm{ml})$ and/or TPM (70 $\mathrm{ng} / \mathrm{ml}$ equi-nicotine units) overnight, then conditional medium was collected. TNF $\alpha$ levels in the conditional medium were analyzed by enzyme-linked immunosorbent assay (ELISA) using ELISA Kit from eBioscience according to the manufacturer's instructions.

\section{Confocal Microscopy}

Neutrophils were treated with PBS, LPS, or TPM overnight, and then fixed with $4 \%$ PFA, deposited on slides through cytospin, and permeabilized with $0.2 \%$ Trinton X-100. The cells were blocked and stained with Alexa Fluor 488 anti-COX4 (NOVUS) antibody and Cy3-anti-LAMP1 antibody (Abcam) in the dark at room temperature. The samples were observed under a confocal microscope.

\section{Statistics}

All experiments were performed at least 3 times. Representative and reproducible results were shown. Statistical analysis was performed with Prism software (GraphPad Software, La Jolla, CA). Values were expressed as means \pm SEM. The significance of the differences was assessed by Student's $t$-test. $P<0.05$ was considered statistically significant.

\section{RESULTS}

\section{Dose Selection of Total Particulate Matter (TPM) for Neutrophil Survival}

In order to optimize our in vitro experimental system for evaluating neutrophil responses to TPM challenge, we first tested the effects of varying dosages of TPM on neutrophil survival 
in vitro. As shown in Figure 1B, compared to vehicle (DMSO) treatment, higher dose of TPM $(1.4 \mu \mathrm{g} / \mathrm{ml}$ equi-nicotine units) resulted in $99 \%$ neutrophils undergoing apoptosis after 1 day culture, suggesting that high dose of TPM $(1.4 \mu \mathrm{g} / \mathrm{ml}$ equinicotine units) is toxic to neutrophils. However, neutrophil survival within both 1 and 3 day culture periods under the treatment of relatively lower doses of TPM $(7$ or $70 \mathrm{ng} / \mathrm{ml}$ equi-nicotine units) were comparable to neutrophils treated with vehicle control. This nanogram-range dosage may also reflect dosages experienced by human smokers (26). Upon optimization of TPM dosages, the non-toxic dose $(70 \mathrm{ng} / \mathrm{ml}$ of equi-nicotine units) of TPM that enabled cell survival was chosen for further examination of neutrophil functional studies.

\section{TPM Suppresses LPS-Induced TNF $\alpha$ Production}

In response to inflammatory signals such as lipopolysaccharide (LPS), neutrophils produce cytokines and chemokines, which play key roles in the regulation of the immune response and mount an effective anti-microbial defense (27). We next tested whether low-dose TPM exposure may affect the expression of selected inflammatory cytokines by neutrophils in response to LPS. To test this, neutrophils were treated with $1 \mu \mathrm{g} / \mathrm{ml} \mathrm{LPS}$, in the presence or absence of TPM, for $4 \mathrm{~h}$ as described in Materials and Methods. Treated cells were stained for intracellular TNF $\alpha$. As a positive control, we observed that LPS treatment alone increased TNF $\alpha$ levels produced by neutrophils (Figures $\mathbf{2 A}, \mathbf{C}$ ). $\mathrm{TPM}$ alone also reduced $\mathrm{TNF} \alpha$ expression in neutrophils.
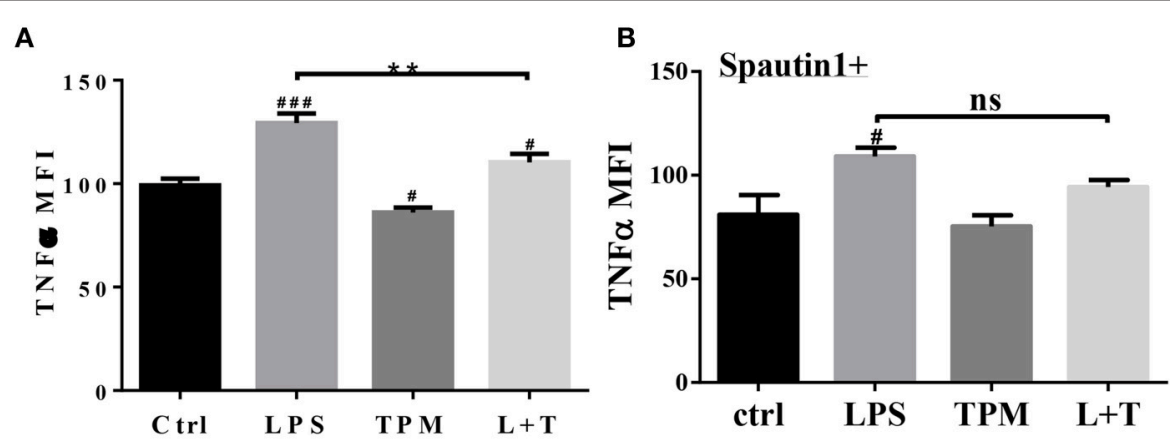

C

D
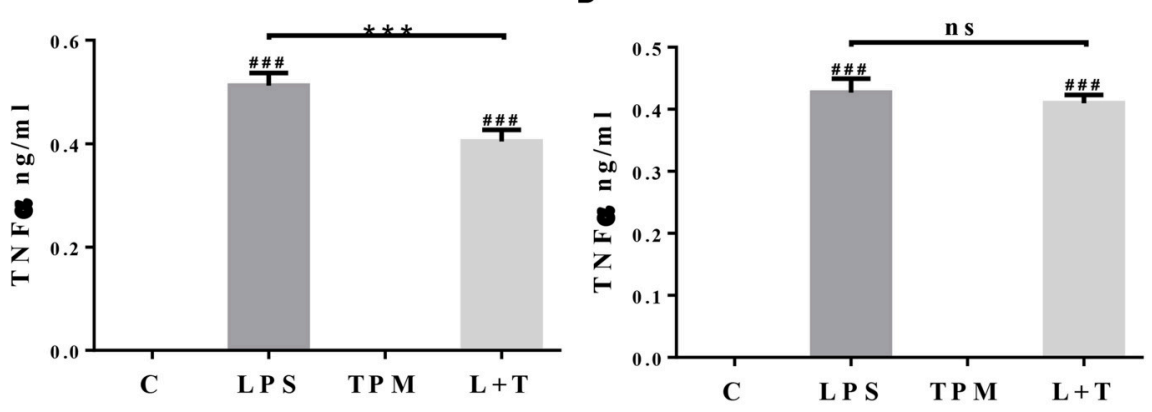

E
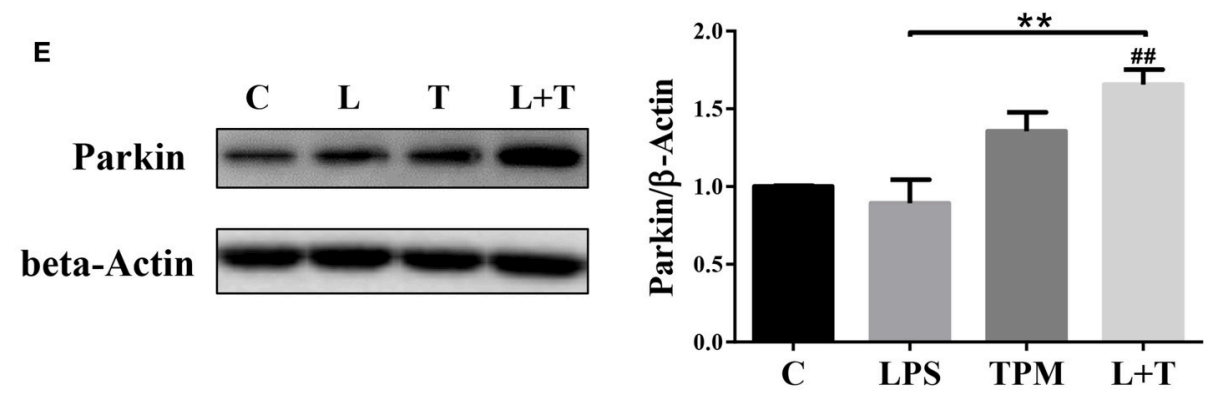

FIGURE 2 | TPM reduced TNF $\alpha$ production by neutrophils induced by LPS. Bone marrow neutrophils were pre-treated without (A) or with autophagy inhibitor Spautin1 (B) $30 \mathrm{~min}$ before stimulation with LPS $(1 \mu \mathrm{g} / \mathrm{ml})$ and/or TPM $(70 \mathrm{ng} / \mathrm{ml}$ equi-nicotine units) for $4 \mathrm{~h}$. Intracellular TNF $\alpha$ levels were measured by flow cytometry analysis. ${ }^{\star *} p<0.01 ;{ }^{*} p<0.05, \# \# \# p<0.001$ as compared with control group. Purified neutrophils were pre-treated without (C) or with autophagy inhibitor Spautin1. ${ }^{* *} \mathrm{p}<0.001$; \#\#\# $\mathrm{p}<0.001$ as compared with control group. (D) $30 \mathrm{~min}$ before stimulation with LPS (100 ng/ml) and/or TPM (70 ng/ml equi-nicotine units) overnight, then conditional medium was collected and subjected to ELISA analysis. (E) Purified neutrophils were treated with LPS (100 ng/ml) and TPM ( $70 \mathrm{ng} / \mathrm{ml}$ equi-nicotine units) overnight, then subjected to immunoblotting. Bar graph on the right panel is the quantification results of protein expression from three experiments, normalized to $\beta$-actin. ${ }^{\star *} p<0.01$; $\# \# p<0.01$ as compared with control group. 
Furthermore, when neutrophils were co-stimulated with TPM and LPS, the levels of TNF $\alpha$ were significantly reduced as compared to cells treated with LPS alone (Figures 2A,C). Our data suggest that TPM is a negative regulator of neutrophil inflammatory activation.

To determine its potential mechanism, we tested whether TPM may attenuate neutrophil inflammatory activation through inducing homeostatic autophagy. Autophagy is involved in reducing cellular stress and is known to be activated by TPM (28). To test whether autophagy is involved in TPM-mediated suppression of TNF $\alpha$ expression, autophagy inhibitor, spautin1, was added to the culture system. As shown in Figures 2B,D, inhibition of autophagy by spautin-1 blocked the TPM effect and restored TNF $\alpha$ induction by LPS in the presence of TPM.

We further measured the levels of Parkin, a molecule involved in the initiation of mitophagy, and observed an induction of Parkin by TPM and LPS co-treatment, indicative of mitophagy activation by TPM (Figure 2E). To further independently confirm that LPS-mediated mitophagy disruption can be remedied by TPM, we performed confocal analyses. Consistent with our Western blot analyses presented above, LPS challenge dramatically disrupted the fusion of mitochondria and lysosome. In contrast, TPM co-treatment restored mitophagy in cells challenged with LPS (Figure 3). Together, our data suggest that TPM reduces LPS-induced TNF $\alpha$ expression through inducing autophagy-mediated homeostasis.

\section{TPM Decreases Neutrophil Bacterial Killing Activity}

Elimination of invading microbes is one of the major functions of neutrophils. Since TPM potentially suppressed neutrophil

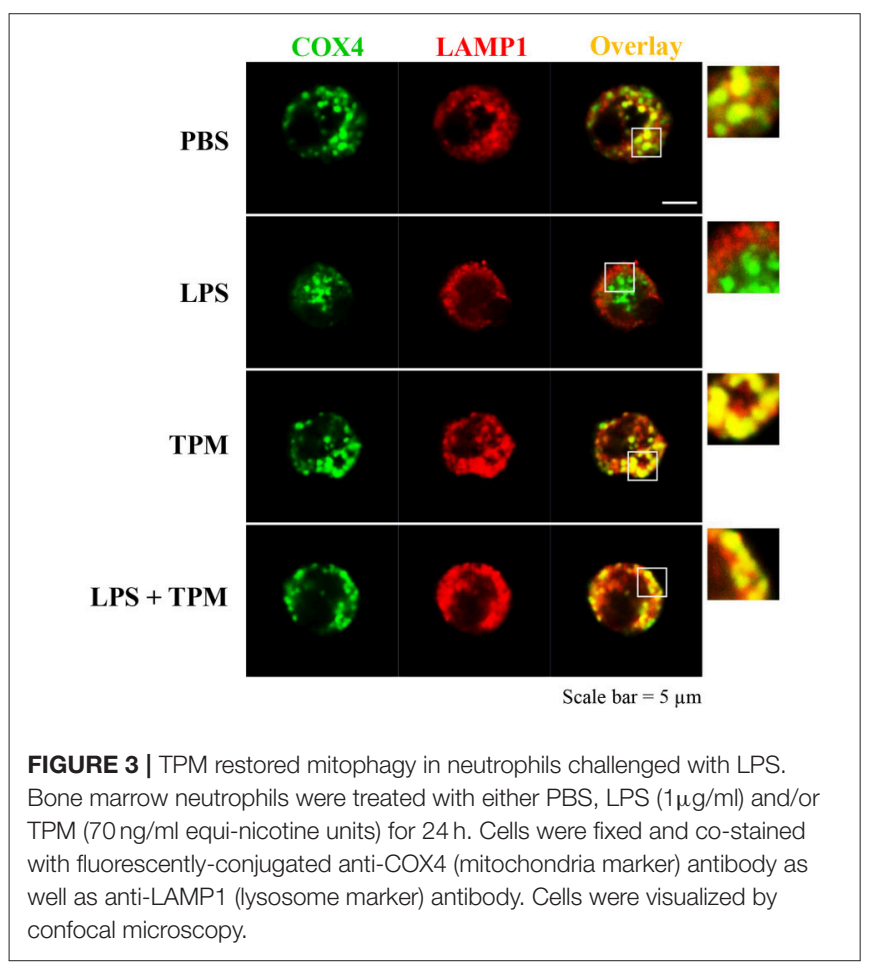

activation in terms of TNF expression, we next studied whether TPM may also compromise the bacterial killing capability of neutrophils. To test this, neutrophils were treated with LPS and/or TPM overnight, then loaded with opsonized E.coli for $1 \mathrm{~h}$, followed by cell lysis. Cell lysates were plated on bacterial growth media, and surviving bacteria colonies were counted the next day. As shown in Figure 4, LPS priming increased neutrophil bactericidal activity, as reflected by lower CFU counting (Figure 4A) and higher bacterial killing rate (Figure 4B). However, in cells pre-treated with both LPS and TPM, CFU counts were significantly elevated as compared to cells pre-treated with LPS alone. Our data show that TPM compromises the bactericidal activity of neutrophils.

\section{TPM Reduces NADPH Oxidase Components and Stat1 Activation}

In order to further determine molecular mechanisms for TPMmediated reduction of neutrophil bacterial killing activity, we tested neutrophil NADPH oxidase components involved in oxidative burst and bacterial killing. Neutrophils were treated with LPS and/or TPM for $24 \mathrm{~h}$. Treated cells were subjected to the measurement of NADP/NADPH levels as described in the Materials and Methods section. The levels of NADP/NADPH
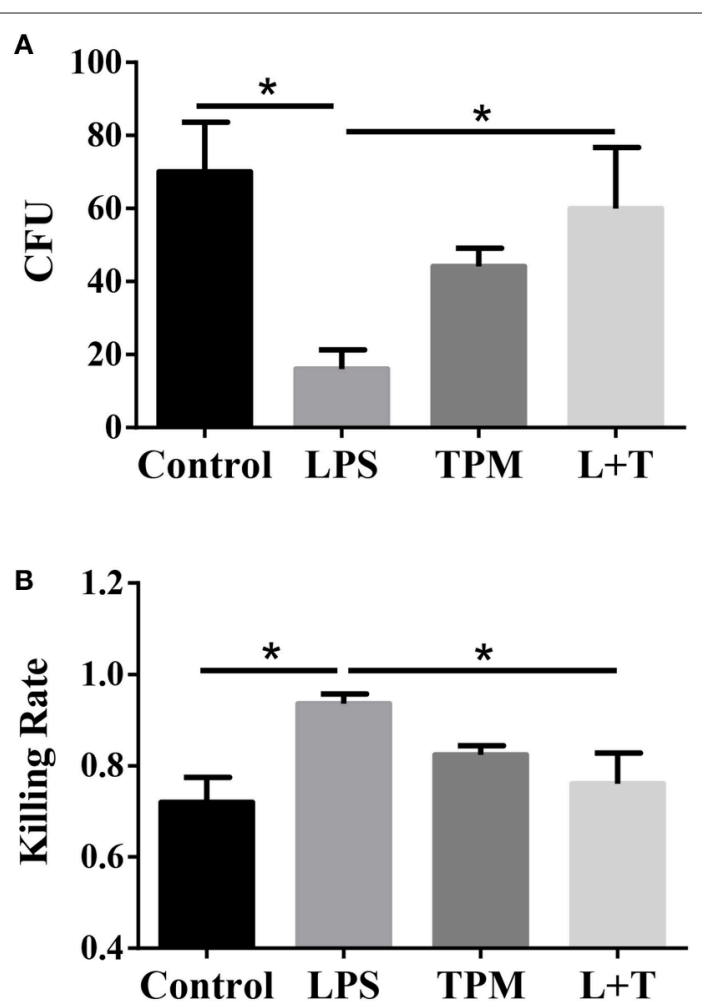

FIGURE 4 | TPM decreased the bacteria killing activity of LPS-primed neutrophils. (A) In vitro bacterial killing by neutrophils primed with LPS and/or TPM. Intra-cellular bacteria harvested from neutrophils were plated on agar plates, incubated overnight, and quantified as bacteria colony-forming units (CFU). (B) Relative bacterial killing rates were calculated according to the input control. Data represent three experiments. ${ }^{*} p<0.05$. 
were increased in neutrophils treated with LPS, and reduced in neutrophils subjected to the co-treatment with LPS and TPM (Figure 5A). Similar results were also observed in neutrophils primed with PMA (Figure 5B). We further examined the levels of NADPH oxidase component gp91 and observed a reduction of gp91 in neutrophils co-stimulated with LPS and TPM. In addition, we also observed that TPM treatment reduced the expression of iNOS induced by LPS (Figure 5C). Together, our data suggest that TPM is a negative regulator of NADPH oxidase in neutrophils and also reduces the expression of iNOS.

Next, we examined the potential molecular mechanisms that underlie the reduced neutrophil activation by TPM treatment. STAT1 has been shown to be critically involved in neutrophil activation and the expression of pro-inflammatory as well as anti-bacterial mediators such as iNOS and gp96 $(29,30)$. We therefore tested the activation status of STAT1 by probing the phosphorylated pSTAT1 levels in neutrophils
A

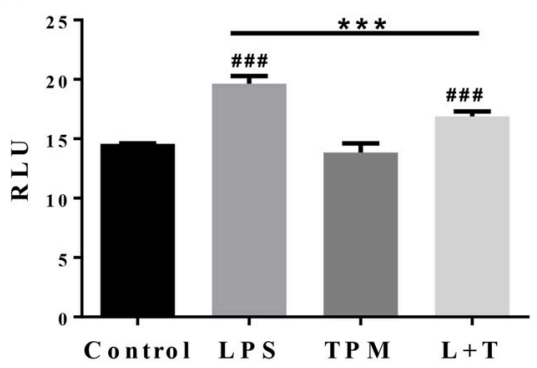

C
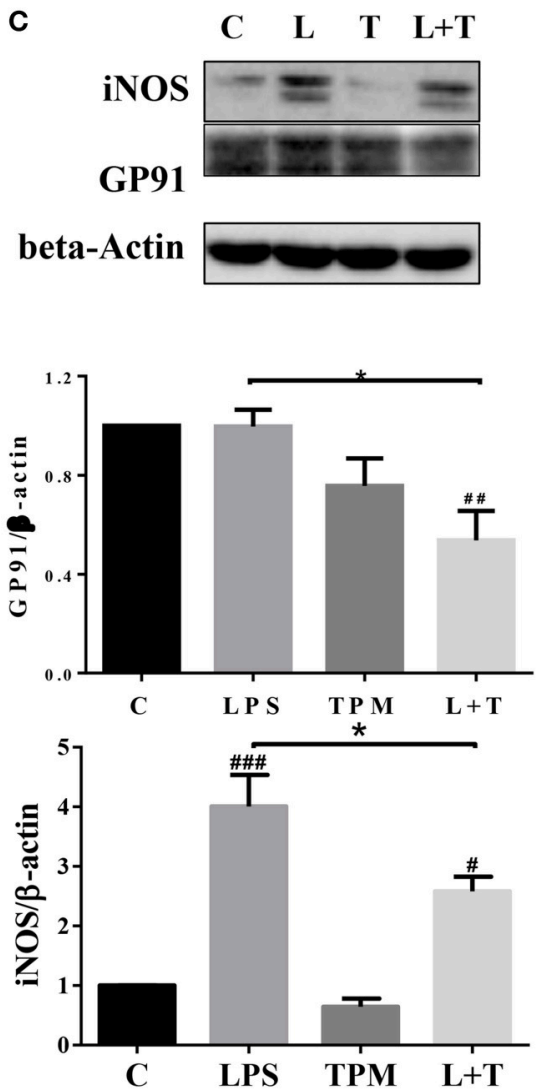

B

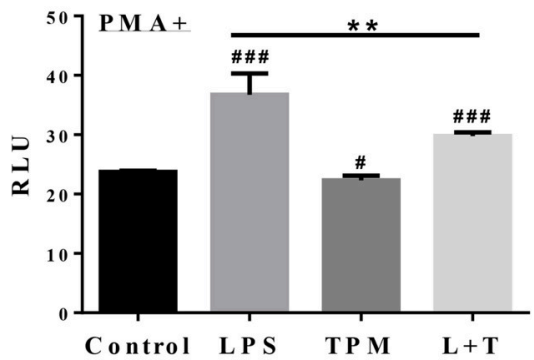

D
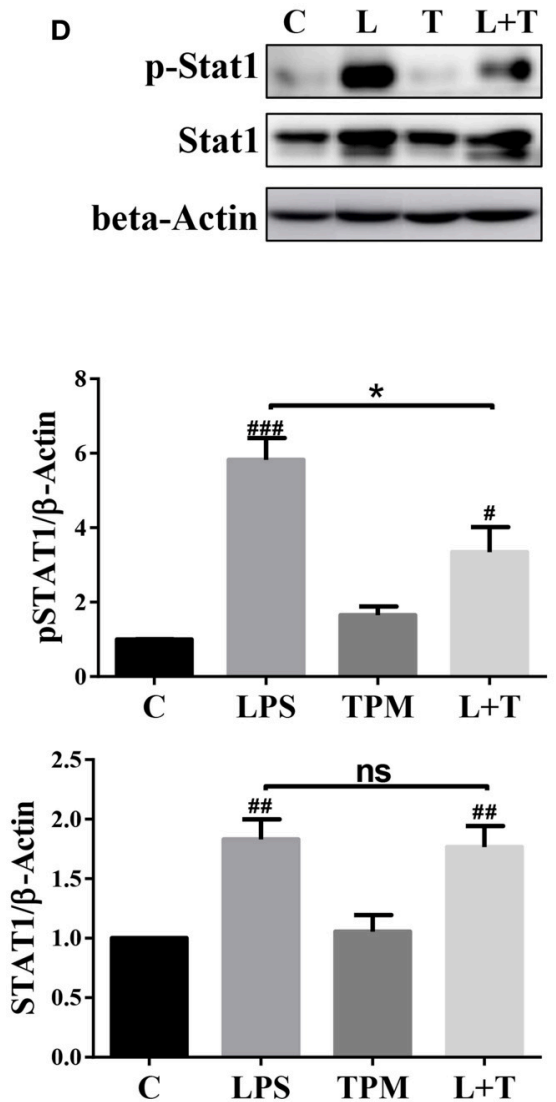

FIGURE 5 | TPM decreased the levels of iNOS, NADPH oxidase component GP91, and STAT1 activation in LPS-primed neutrophils. Purified neutrophils were treated with LPS $(100 \mathrm{ng} / \mathrm{ml})$ and/or TPM $(70 \mathrm{ng} / \mathrm{ml}$ equi-nicotine units) overnight, followed without (A) or with (B) stimulation of PMA (20 ng/ml) for $15 \mathrm{~min}$, then subjected to NADP/NADPH measurement. ${ }^{* *} p<0.01,{ }^{* * *} p<0.001 ; \# p<0.05, \# \# \# p<0.001$ compared with control group. (C,D) Purified neutrophils were treated with LPS $(100 \mathrm{ng} / \mathrm{ml})$ and/or TPM $(70 \mathrm{ng} / \mathrm{ml}$ equi-nicotine units) overnight, then subjected to immunoblotting to measure the levels of iNOS, gp91, total STAT1, and p-STAT1. Data represent three experiments. Bar graphs on the bottom panels are the quantification results of protein expressions, normalized to $\beta$-actin. ${ }^{*} p<0.05$; $\# p<0.05$, $\# \# p<0.01$ compared with control group. 
treated with LPS and/or TPM. As shown in Figure 5D, LPS significantly induced the pSTAT1 levels in neutrophils. TPM alone has no effect on neutrophil pSTAT1 levels. Cotreatment with TPM and LPS attenuated the neutrophil pSTAT1 levels induced by LPS. Our data reveal that TPM may contribute to compromised neutrophil bacterial killing activity by reducing STAT1-mediated expression of NADPH oxidase and iNOS.

\section{DISCUSSION}

The results reported in this manuscript indicate that lowdose cigarette smoke TPM compromises the anti-microbial defense function of neutrophils. Several lines of evidence support our conclusion. First, we observed that neutrophils treated with low-dose TPM demonstrate reduced bacterial killing activity without apparent alteration in neutrophil viability. Second, we documented that TPM-treated neutrophils have reduced capacity to express inflammatory cytokines, reduced bacterial killing activity, reduced expression of iNOS, and reduced NADPH oxidase components. Third, TPM-treated neutrophils have reduced activation of STAT1, a key transcription factor involved in the expression of iNOS and key NADPH oxidase components such as gp91.

At the translational level, our data provide a conceptual advance in terms of how TPM may compromise host immune environment and contribute to chronic health conditions observed in smokers. Past studies regarding TPM on immune cells largely focused on the high dosages of TPM on cellular survival and death $(22,31)$. Although these studies are important and may reveal the highly toxic effect of smoking, they may not fully reflect the chronic effects of regular smoking with low-levels exposure. Our data reveal that low-levels of TPM exposure is sufficient to compromise neutrophil functions without apparent cell death. Neutrophils are predominant innate leukocytes in the host lung and related mucosal tissues. Proper functions of neutrophils are essential for maintaining proper host defense toward infectious and inflammatory signals (32). Compromised neutrophil antimicrobial function may subject the host to higher risks of chronic infection, as often seen in humans with chronic smoking (33, 34).

Our data also provide additional support for the emerging concept of innate immune programming and memory. Although previous dogma claim that innate leukocytes such as monocytes and neutrophils are first responders to challenges with limited modulatory roles nor memory, recent studies suggest that more complex programming and memory dynamics of neutrophils may exist (35). For example, tumor-infiltrating neutrophils tend to adopt an anti-inflammatory state with elevated expression of TGF $\beta$ (36). Humans with chronic alcohol consumption tend to have neutrophils with reduced anti-microbial functions (37). Consequently, reprogrammed neutrophils with altered functions may pre-dispose the host for the pathogenesis of chronic diseases. Our current data lend further support for this concept, and reveal that TPM from cigarette smoke may program neutrophils into an immune-compromised state with reduced anti-microbial capability. Our data lend mechanistic support for previous epidemiology observations that document increased risks for microbial infections in chronic smokers (38-40). Chronic smokers were known to exhibit elevated risks, severities with increased relapse, and mortality associated with tuberculosis (38). Both direct and second-hand smoke exposure are also reported to be associated with higher risks of lung microbial infections causing pneumococcus meningitis (39). Many of the adverse effects such as COPD related with chronic smoking may linger long after smoking cessation (40). Reprogrammed neutrophils with compromised antimicrobial activity due to TPM challenge as we reported herein may underlie elevated risks of chronic infection and lung pathology.

At the molecular level, our data reveal that TPM skews neutrophil function through reducing STAT1-mediated expression of inflammatory mediators as well as critical antibacterial components such as iNOS and NADPH oxidase components, both of which are important for the antibacterial function of neutrophils $(41,42)$. Our data also reveal that TPM may reduce neutrophil inflammatory activation through inducing mitophagy. Further mechanistic studies are warranted to dissect the detailed mechanisms that underlie the regulation of neutrophil homeostasis by TPM. Collectively, our study has revealed an important modulatory role of TPM in compromising the anti-bacterial killing function of neutrophils.

\section{ETHICS STATEMENT}

This study was carried out in accordance with the recommendations of Virginia Tech Institutional Guideline for proper usage of animals. The protocol was approved by the Institutional Animal Care and Usage Committee of Virginia Tech.

\section{AUTHOR CONTRIBUTIONS}

YZ conducted the experiment, analyzed the data, and wrote the manuscript. SG conducted the experiment and analyzed the data. GP analyzed the data and wrote the manuscript. LL designed the study, analyzed the data, and wrote the manuscript.

\section{FUNDING}

This work is funded through a postdoctoral fellowship program by R. J. Reynolds Tobacco Company, a wholly owned subsidiary of Reynolds American Inc., which is a wholly owned subsidiary of British American Tobacco plc.

\section{ACKNOWLEDGMENTS}

We would like to thank members of the $\mathrm{Li}$ lab in helpful discussion and technical assistance. 


\section{REFERENCES}

1. Stampfli MR, Anderson GP. How cigarette smoke skews immune responses to promote infection, lung disease and cancer. Nat Rev Immunol. (2009) 9:377-84. doi: 10.1038/nri2530

2. Sopori M. Effects of cigarette smoke on the immune system. Nat Rev Immunol. (2002) 2:372-7. doi: 10.1038/nri803

3. Motz GT, Eppert BL, Sun G, Wesselkamper SC, Linke MJ, Deka R, et al. Persistence of lung CD8 T cell oligoclonal expansions upon smoking cessation in a mouse model of cigarette smoke-induced emphysema. J Immunol. (2008) 181:8036-43. doi: 10.4049/jimmunol.181.1 1.8036

4. Maeno T, Houghton AM, Quintero PA, Grumelli S, Owen CA, Shapiro $\mathrm{SD}$. $\mathrm{CD}^{+} \mathrm{T}$ Cells are required for inflammation and destruction in cigarette smoke-induced emphysema in mice. J Immunol. (2007) 178:8090-6. doi: 10.4049/jimmunol.178.12.8090

5. Trimble NJ, Botelho FM, Bauer CM, Fattouh R, Stampfli MR. Adjuvant and anti-inflammatory properties of cigarette smoke in murine allergic airway inflammation. Am J Respir Cell Mol Biol. (2009) 40:38-46. doi: 10.1165/rcmb.2008-0107OC

6. Paget C, Trottein F. Role of type 1 natural killer $\mathrm{T}$ cells in pulmonary immunity. Mucosal Immunol. (2013) 6:1054-67. doi: 10.1038/mi.2 013.59

7. Culley FJ. Natural killer cells in infection and inflammation of the lung. Immunology (2009) 128:151-63. doi: 10.1111/j.1365-2567.2009.0 3167.x

8. King TE Jr, Savici D, Campbell PA. Phagocytosis and killing of Listeria monocytogenes by alveolar macrophages: smokers versus nonsmokers. J Infect Dis. (1988) 158:1309-16.

9. Tollerud DJ, Clark JW, Brown LM, Neuland CY, Mann DL, PankiwTrost LK, et al. Association of cigarette smoking with decreased numbers of circulating natural killer cells. Am Rev Respir Dis. (1989) 139: 194-8.

10. Zappacosta B, Persichilli S, Minucci A, Fasanella S, Scribano D, Giardina B, et al. Effect of cigarette smoke extract on the polymorphonuclear leukocytes chemiluminescence: influence of a filter containing glutathione. Luminescence (2005) 20:73-5. doi: 10.1002/bio.805

11. Marti-Lliteras P, Regueiro V, Morey P, Hood DW, Saus C, Sauleda J, et al. Nontypeable Haemophilus influenzae clearance by alveolar macrophages is impaired by exposure to cigarette smoke. Infect Immun. (2009) 77:4232-42. doi: 10.1128/IAI.00305-09

12. Hodge S, Matthews G, Mukaro V, Ahern J, Shivam A, Hodge G, et al. Cigarette smoke-induced changes to alveolar macrophage phenotype and function are improved by treatment with procysteine. Am J Respir Cell Mol Biol. (2011) 44:673-81. doi: 10.1165/rcmb.2009-0459OC

13. Hodge S, Hodge G, Ahern J, Jersmann H, Holmes M, Reynolds PN. Smoking alters alveolar macrophage recognition and phagocytic ability: implications in chronic obstructive pulmonary disease. Am J Respir Cell Mol Biol. (2007) 37:748-55. doi: 10.1165/rcmb.2007-0 025OC

14. Rosendahl A, Bergmann S, Hammerschmidt S, Goldmann O, Medina E. Lung dendritic cells facilitate extrapulmonary bacterial dissemination during pneumococcal pneumonia. Front Cell Infect Microbiol. (2013) 3:21. doi: $10.3389 /$ fcimb.2013.00021

15. MacNee W. Pathogenesis of chronic obstructive pulmonary disease. Proc Am Thorac Soc. (2005) 2:258-66. doi: 10.1513/pats.200504045SR

16. Pitzer JE, Del Zoppo GJ, Schmid-Schonbein GW. Neutrophil activation in smokers. Biorheology (1996) 33:45-58.

17. Overbeek SA, Braber S, Koelink PJ, Henricks PA, Mortaz E, LoTam Loi AT, et al. Cigarette smoke-induced collagen destruction; key to chronic neutrophilic airway inflammation? PLOS ONE (2013) 8:e55612. doi: 10.1371/journal.pone.0055612

18. Thornton WH Jr, Adelstein EH, Edes TE. Leukotriene B4 is measurable in serum of smokers and nonsmokers. Clin Chem. (1989) 35: 459-60.

19. Afonso PV, Janka-Junttila M, Lee YJ, McCann CP, Oliver CM, Aamer KA, et al. LTB4 is a signal-relay molecule during neutrophil chemotaxis. Dev Cell (2012) 22:1079-91. doi: 10.1016/j.devcel.2012. 02.003

20. Stempelj M, Kedinger M, Augenlicht L, Klampfer L. Essential role of the JAK/STAT1 signaling pathway in the expression of inducible nitric-oxide synthase in intestinal epithelial cells and its regulation by butyrate. J Biol Chem. (2007) 282:9797-804. doi: 10.1074/jbc.M6094 26200

21. Kumatori A, Yang D, Suzuki S, Nakamura M. Cooperation of STAT-1 and IRF-1 in interferon-gamma-induced transcription of the gp91(phox) gene. J Biol Chem. (2002) 277:9103-11. doi: 10.1074/jbc.M1098 03200

22. Arimilli S, Damratoski BE, Bombick B, Borgerding MF, Prasad GL. Evaluation of cytotoxicity of different tobacco product preparations. Regul Toxicol Pharmacol. (2012) 64:350-60. doi: 10.1016/j.yrtph.2012. 09.004

23. Diao N, Zhang Y, Chen K, Yuan R, Lee C, Geng S, et al. Deficiency in Toll-interacting protein (Tollip) skews inflamed yet incompetent innate leukocytes in vivo during DSS-induced septic colitis. Sci Rep. (2016) 6:34672. doi: $10.1038 /$ srep 34672

24. Green JN, Winterbourn CC, Hampton MB. Analysis of neutrophil bactericidal activity. Methods Mol Biol. (2007) 412:319-32. doi: 10.1007/978-1-59745-467-4_21

25. Geng S, Chen K, Yuan R, Peng L, Maitra U, Diao N, et al. The persistence of low-grade inflammatory monocytes contributes to aggravated atherosclerosis. Nat Commun. (2016) 7:13436. doi: 10.1038/ncomms 13436

26. Robinson RJ, $\mathrm{Yu}$ CP. Deposition of cigarette smoke particles in the human respiratory tract. Aerosol Sci Technol. (2001) 34:202-15. doi: $10.1080 / 027868201300034844$

27. Tecchio C, Micheletti A, Cassatella MA. Neutrophil-derived cytokines: facts beyond expression. Front Immunol. (2014) 5:508. doi: 10.3389/fimmu.2014.00508

28. Zhou JS, Zhao Y, Zhou HB, Wang Y, Wu YF, Li ZY, et al. Autophagy plays an essential role in cigarette smoke-induced expression of MUC5AC in airway epithelium. Am J Physiol Lung Cell Mol Physiol. (2016) 310:L1042-52. doi: 10.1152/ajplung.00418.2015

29. Luu K, Greenhill CJ, Majoros A, Decker T, Jenkins BJ, Mansell A. STAT1 plays a role in TLR signal transduction and inflammatory responses. Immunol Cell Biol. (2014) 92:761-9. doi: 10.1038/icb.2014.51

30. Sun B, Hu X, Liu G, Ma B, Xu Y, Yang T, et al. Phosphatase Wipl negatively regulates neutrophil migration and inflammation. J Immunol. (2014) 192:1184-95. doi: 10.4049/jimmunol.13 00656

31. Massarsky A, Jayasundara N, Bailey JM, Oliveri AN, Levin ED, Prasad GL, et al. Teratogenic, bioenergetic, and behavioral effects of exposure to total particulate matter on early development of zebrafish (Danio rerio) are not mimicked by nicotine. Neurotoxicol Teratol. (2015) 51:77-88. doi: 10.1016/j.ntt.2015.09.006

32. Kolaczkowska E, Kubes P. Neutrophil recruitment and function in health and inflammation. Nat Rev Immunol. (2013) 13:159-75. doi: 10.1038/nr i3399

33. Arnson Y, Shoenfeld Y, Amital H. Effects of tobacco smoke on immunity, inflammation and autoimmunity. J Autoimmun. (2010) 34:J258-65. doi: 10.1016/j.jaut.2009.12.003

34. Lugade AA, Bogner PN, Thatcher TH, Sime PJ, Phipps RP, Thanavala Y. Cigarette smoke exposure exacerbates lung inflammation and compromises immunity to bacterial infection. J Immunol. (2014) 192:5226-35. doi: 10.4049/jimmunol.1302584

35. Lee C, Geng S, Zhang Y, Rahtes A, Li L. Programming and memory dynamics of innate leukocytes during tissue homeostasis and inflammation. J Leukoc Biol. (2017) 102:719-26. doi: 10.1189/jlb.6MR0117027RR

36. Fridlender ZG, Sun J, Kim S, Kapoor V, Cheng G, Ling L, et al. Polarization of tumor-associated neutrophil phenotype by TGF-beta: "N1" versus “N2" TAN. Cancer Cell (2009) 16:183-94. doi: 10.1016/j.ccr.2009. 06.017

37. Simet SM, Sisson JH. Alcohol's effects on lung health and immunity. Alcohol Res. (2015) 37:199-208. 
38. Altet-Gomez MN, Alcaide J, Godoy P, Romero MA, Hernandez del Rey I. Clinical and epidemiological aspects of smoking and tuberculosis: a study of 13,038 cases. Int J Tuberc Lung Dis. (2005) 9:430-6.

39. Gold R. Epidemiology of bacterial meningitis. Infect Dis Clin North Am. (1999) 13:515-25. doi: 10.1016/S0891-5520(05)7 0092-1

40. Hogg JC. Why does airway inflammation persist after the smoking stops? Thorax (2006) 61:96-7. doi: 10.1136/thx.2005.049502

41. Van Avondt K, van Sorge NM, Meyaard L. Bacterial immune evasion through manipulation of host inhibitory immune signaling. PLoS Pathog. (2015) 11:e1004644. doi: 10.1371/journal.ppat.10 04644

42. El-Benna J, Hurtado-Nedelec M, Marzaioli V, Marie JC, GougerotPocidalo MA, Dang PM. Priming of the neutrophil respiratory burst: role in host defense and inflammation. Immunol Rev. (2016) 273:180-93. doi: $10.1111 / \mathrm{imr} .12447$
Conflict of Interest Statement: GP, is a full time employee of RAI Services Company. RAI Services Company is a wholly owned subsidiary of Reynolds American Inc., which is a wholly owned subsidiary of British American Tobacco plc.

The remaining authors declare that the research was conducted in the absence of any commercial or financial relationships that could be construed as a potential conflict of interest.

Copyright (c) 2018 Zhang, Geng, Prasad and Li. This is an open-access article distributed under the terms of the Creative Commons Attribution License (CC BY).

The use, distribution or reproduction in other forums is permitted, provided the original author(s) and the copyright owner(s) are credited and that the original publication in this journal is cited, in accordance with accepted academic practice. No use, distribution or reproduction is permitted which does not comply with these terms. 